\title{
Wirkungen von Fortbildungen zur Sprachförderung für pädagogische Fach- und Lehrkräfte
}

\author{
Karin Kämpfe • Tanja Betz • Diemut Kucharz
}

Eingegangen: 26. Oktober 2020 / Überarbeitet: 14. Mai 2021 / Angenommen: 8. Juni 2021 / Online publiziert: 23. Juli 2021

(C) Der/die Autor(en) 2021

Zusammenfassung In Kindertageseinrichtungen und Schulen wird dem pädagogischen Personal in Bezug auf Sprachförderung ein hoher Qualifizierungsbedarf attestiert; zugleich gibt es wenig gesichertes empirisches Wissen über die Wirkungen entsprechender Fortbildungen. Das Fortbildungsangebot und die Teilnehmenden sind heterogen. Um die Heterogenität der Teilnehmenden zu entschlüsseln, wurden im BiSS-Evaluationsprojekt „,SPRÜNGE“ (Sprachförderung im Übergang Kindergarten - Grundschule evaluieren) auf Grundlage einer Typenbildung von $N=27$ Fachund Lehrkräften Unterschiede in ihren Sprachförderkompetenzen und im Fortbildungsbesuch empirisch-quantitativ mittels des Online-Tests SprachKoPF überprüft und für zwei Sprachfördertypen ihre handlungsleitenden Orientierungen in Bezug auf die Wirkungen von Fortbildungen rekonstruiert. Bei diesen beiden, stark fortgebildeten Sprachfördertypen konnten differenzierbare Deutungen zu den Wirkungen von Fortbildungen herausgearbeitet werden, wobei einerseits eine stärkere KitaOrientierung und andererseits eine stärkere Schulorientierung als (implizite) Referenz deutlich wird. Zudem ließen sich Gelingensbedingungen von Fortbildungen wie „Praxisrelevanz“, die als allgemeingültig angenommen werden, typenspezifisch ausdifferenzieren. Mit der multiperspektivischen Untersuchung der differentiellen Wirkungen von Fortbildungen leistet das Projekt einen explorativen Beitrag zu ei-

Dr. Karin Kämpfe $(\bowtie) \cdot$ Prof. Dr. Tanja Betz

FB 02 - Institut für Erziehungswissenschaft, Allgemeine Erziehungswissenschaft

mit dem Schwerpunkt Kindheitsforschung, Johannes Gutenberg-Universität Mainz,

Jakob-Welder-Weg 12, 55128 Mainz, Deutschland

E-Mail: kaempfe@uni-mainz.de

Prof. Dr. Tanja Betz

E-Mail: tbetz@uni-mainz.de

Prof. Dr. Diemut Kucharz

$\mathrm{Fb} 04$ Erziehungswissenschaften, Institut für Pädagogik der Elementar- und Primarstufe,

Goethe-Universität Frankfurt, Theodor-W.-Adorno-Platz 6, 60629 Frankfurt am Main, Deutschland

E-Mail: kucharz@em.uni-frankfurt.de 
ner quantitativ und qualitativ ausgerichteten Wirkungsforschung am Übergang KitaGrundschule.

Schlüsselwörter Sprachförderung am Übergang Kindergarten-Grundschule · Fortbildungen · Sprachförderkompetenzen · Handlungsleitende Orientierungen · Wirkungsforschung

\title{
Effects of professional development in language support for ECEC professionals and teachers
}

\begin{abstract}
In kindergarten and school, pedagogical staff is considered to have a high need for qualification in language support; at the same time, there is hardly any empirical knowledge about the effectiveness of such professional development. Both the professional development offered and the participants are heterogeneous. In order to decipher the heterogeneity of the participants, the BiSS evaluation project "SPRÜNGE" used the online test SprachKoPF to empirically-quantitatively examine differences in their language support skills and in their attendance at professional development on the basis of a typology of $N=27$ ECEC staff and teachers. Second, for two language support types, their action-guiding orientations regarding the effects of professional development were reconstructed. In the case of these two highly trained language support types, differentiable interpretations with regard to the effects of professional development could be worked out, with a stronger kindergarten orientation on the one hand and a stronger school orientation on the other as an (implicit) reference. In addition, conditions for the success of professional development, such as "practical relevance", which are assumed to be generally valid, can be differentiated according to type. With the multi-perspectival investigation of the differential effects of professional development, the project makes an explorative contribution to quantitative and qualitative impact research at the transition from kindergarten to primary school.
\end{abstract}

Keywords Language support at the transition from kindergarten to primary school · Professional development · Language support skills · Action-guiding orientations · Impact research

\section{Einleitung und Vorstellung der Forschungsfrage}

Unstrittig ist, dass die Kompetenzen von pädagogischen Fach- und Lehrkräften eine wichtige Voraussetzung für eine gelingende Sprachförderung im Sinne der Entwicklung von Kindern darstellen. Im fachlichen Diskurs besteht ebenfalls Einigkeit darüber, dass es einen hohen Qualifizierungsbedarf bei Fach- und Lehrkräften u. a. im Bereich Sprachförderung gibt und berufliche Fortbildungen deshalb wesentlich sind (Von Hippel 2011). Zugleich gilt es auch international als unstrittig, dass Fortbildungen des pädagogischen Personals stark mit der Qualität pädagogischer Einrichtungen zusammenhängen. 
Problematisiert wird hingegen, dass umfassendere empirische Einblicke in die Wirksamkeit von Fortbildungen generell (Schrader et al. 2019) sowie im Kontext Sprachförderung im Besonderen fehlen. Qualifizierungsmaßnahmen im Bereich Sprache gelte es wissenschaftlich und evaluativ besser abzusichern, denn, so Kammermeyer (2019, S. 231), ,über die Qualität der Fortbildung [...] wissen wir in der Regel nichts“. Dies hängt auch damit zusammen, dass ein „Höchstmaß an Heterogenität der Angebote“ zu verzeichnen ist, wie dies Redder et al. (2011, S. 63) in ihrer Bilanz über strukturierte Forschung zu Sprachdiagnostik und -förderung konstatieren. Wenngleich nach Stamm (2014) die anglo-amerikanische Fortbildungsforschung im Bereich früher Kindheit umfangreicher ist als die deutschsprachige Forschung, so gebe es auch dort ,bemerkenswert wenig Befunde zu den Fragen (...), welches denn die wirksamsten Typen professioneller Entwicklung sind und unter welchen Bedingungen ein Mehr an Aus- und Weiterbildung auch $\mathrm{zu}$ besseren kindlichen Entwicklungsergebnissen führt" (Stamm 2014, S. 124f.).

Angesichts dieser ernüchternden Einschätzungen und zugleich der hohen gesellschaftlichen Relevanz früher Sprachförderung und einer durchgängigen sprachlichen Bildung, rückt die Qualifizierung des pädagogischen Personals immer stärker in den Fokus nicht nur von Bildungsadministration und -politik, sondern ebenso der Bildungsforschung. Davon zeugen groß angelegte Entwicklungs- und Forschungsprogramme, Bestandsaufnahmen sowie Qualifizierungsinitiativen (zu einer Übersicht: Redder et al. 2011; Schneider et al. 2012) und entsprechende forschungsorientierte Schriften auch in Form von Buchreihen und Themenheften in Fachzeitschriften, die in jüngster Zeit entstanden sind (u.a. die neue Reihe „Bildung durch Sprache und Schrift" (Band 1-5), das Heft der Zeitschrift Empirische Pädagogik von 2018 „Sprachförderung: Formative und summative (BiSS-) Evaluationen im Elementarund Primarbereich“ und das Heft „Bildung durch Sprache und Schrift“ der Zeitschrift Frühe Bildung von 2019). Gleichwohl zeigen sich auch mit zunehmender Forschungsintensität markante Desiderate. Hierzu gehört Forschung zu den an der Sprachförderung beteiligten Pädagog*innen an Übergängen zwischen den Systemen Kita-Grundschule, da bisherige Studien zum pädagogischen Personal zumeist trennen zwischen der ,frühen Bildung“ einerseits und der „Schule“ andererseits (exemplarisch: Schrader et al. 2019). Zudem gibt es zahlreiche quantitative Forschungsprojekte, die Zusammenhänge zwischen (sprachbezogenen) Fortbildungen und (sprachförderlichem) Wissen und Können und bisweilen im Handeln nachweisen können (z. B. Kammermeyer et al. 2019). Wie diese komplexen (Wirkungs-)Zusammenhänge auch angesichts der Heterogenität des pädagogischen Personals zustande kommen, dazu liegt noch wenig Wissen vor. Qualitative Zugänge, die bisher selten zum Einsatz kommen, können über Typologien (Schmidt-Hertha und Tippelt 2011) zu einem besseren Verstehen der (Wirkungs-)Zusammenhänge beitragen, indem sie Strukturen und Ordnungsmuster aufzudecken erlauben, die das (sprachförderliche) Handeln der Pädagog*innen fundieren. Diesen Desideraten nimmt sich der vorliegende Beitrag an. 


\section{Theoretischer Rahmen, Forschungslage und Entwicklung der Fragestellungen}

\subsection{Theoretischer Rahmen}

Einhergehend mit Reformen, u.a. am Übergang in die Grundschule, steigen die Erwartungen an die Leistungen des Systems bzw. an die Kopplung der Systeme und an das pädagogische Personal (Betz 2013; Betz et al. 2016). Dies gilt auch für die Sprachförderung bei Kindern (u.a. Schneider et al. 2012) und die ,durchgängige Sprachbildung“, u.a. verstanden als ein spezifisches Kooperationserfordernis am Übergang zwischen Kindertageseinrichtung und Schule (Salem 2018).

An den gestiegenen Anforderungen und Erwartungen an das pädagogische Personal setzen Kompetenzmodelle an, die das professionelle pädagogische Handeln kompetenztheoretisch beschreiben und erklären und insgesamt sowohl im Kontext Kindertageseinrichtungen als auch im Kontext Schule weit verbreitet sind. Trotz einiger Unterschiede im Detail (hierzu u. a. Baumert und Kunter 2006; Fröhlich-Gildhoff et al. 2011), gehen alle Modelle davon aus, dass professionelle Kompetenzen durch Wissens- und Könnensfacetten sowie motivationale Aspekte beeinflusst werden, ebenso wie durch die Überzeugungen/Beliefs oder die Orientierungen/Haltung des pädagogischen Personals, die wiederum Einfluss auf das Handeln in einer konkreten Situation haben. Zugleich liegt den Modellen die Annahme zugrunde, dass sich Bedingungen der gezielten Entwicklung und Förderung von Wissen und Können und der Beeinflussbarkeit von motivationalen Faktoren und Überzeugungen sowie der Haltung über Fortbildungen schaffen lassen (hierzu u. a. die kompetenzorientierte Weiterbildungsinitiative für frühpädagogische Fachkräfte WiFF; internationale Meta-Analysen z. B. Fukkink und Lont 2007; Markussen-Brown et al. 2017). Entsprechend unterschiedlicher theoretischer Grundlagen, wird in den Modellen auf

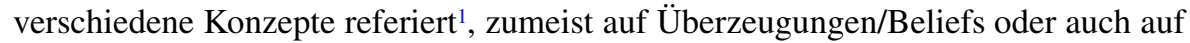
Orientierungen/Haltung. Folgend kommt ihnen eine unterschiedliche Bedeutung in der Modellierung zu (s. unten).

Bezogen auf den Bereich Sprache existieren spezifischere sprachwissenschaftlich fundierte Kompetenzmodelle, die sich, wie bei Hopp et al. (2010), unterteilen lassen in bereichsbezogene Kenntnisse (Wissen), Fähigkeiten (Können) und Handlungen (Machen). Sprachförderkompetenz wird definiert als die Kompetenz, im pädagogischen Alltag Sprachfördersituationen zu schaffen, die auf den Entwicklungsstand des Kindes abgestimmt sind und die Bedingungen des natürlichen Spracherwerbs emulieren (Hopp et al. 2010, S. 613 ff.). Entsprechend fließen die Faktoren z. B. in Qualitätsanforderungen für die Aus- und Fortbildung der Fachkräfte ein.

\footnotetext{
1 In Modellen, in Studien und (internationalen) Metaanalysen werden die Konzepte Überzeugungen, Werthaltungen, Einstellungen, Meinungen, beliefs und attitudes, teilweise synonym, teilweise differenzierend verwendet. Vielfach wird auf Überzeugungen, verstanden als Meinungen und Bewertungen, Bezug genommen (u. a. Kunter und Pohlmann 2015) oder auf die Haltung, verstanden als Habitus (u. a. Fröhlich-Gildhoff et al. 2011) und damit zugleich stärker als modus operandi, referiert. Andernorts werden Überzeugungen wiederum als „Bestandteil der professionellen Haltung“ konzipiert (z. B. Kurucz et al. 2020, S. 715). Eine eineindeutige Zuordnung, welche theoretischen Bezüge diesen Konzepten v. a. mit Blick auf die Analysen jeweils zugrunde liegen, ist zumeist nicht möglich und bedarf weiterer theoretischer Klärungen.
} 
Das Qualifizierungsangebot im Bereich Sprache und Sprachförderung ist äußerst vielfältig (Kucharz 2018). Es unterscheidet sich sowohl im Umfang (von zweistündigen bis zu neuntägigen, auf mehrere Monate verteilten Fortbildungen), in den Anbietern als auch in den Inhalten. Grob lassen sich drei Richtungen unterscheiden: eher sprachwissenschaftlich, pädagogisch oder psychologisch orientierte Fortbildungen (Überblick bei Kucharz 2018). Der größte Teil länger dauernder Fortbildungsreihen vereint mehrere Aspekte. Folgende Themenbereiche sind über die drei Richtungen hinweg relevant: Grundlagen der deutschen Sprache (grammatikalische Strukturen, Lexik/Semantik und Phonologie), Erst- und Zweitspracherwerb sowie Mehrsprachigkeit, Verfahren zur Sprachdiagnostik sowie Planung und Durchführung von Sprachförderung (Kucharz 2018, S. 252). Ergänzt werden oft Themen wie Elternarbeit und Literacy bzw. Bildungssprache.

Trotz Begriffsklärungen, Systematisierungen und Modellbildungen ist nach wie vor offen, wie es gelingen kann, die als erforderlich erachteten (sprachförderlichen) Kompetenzen zu entwickeln, zu fördern und wie genau diese in die eigene Handlungspraxis transferiert werden. Hier setzen (erweiterte) Angebots-Nutzungsmodelle an. Auch sie sind heterogen und zugleich weit verbreitet (zu einer Übersicht: Kohler und Wacker 2013), werden aber nicht spezifisch auf den Kontext Sprachförderung bezogen. Im Hinblick auf Fortbildungen (primär von Lehrkräften: Lipowsky und Rzejak 2018) werden nicht nur ,,angebotsseitig“ die Kompetenzen der Fortbildner*innen fokussiert, sondern gleichzeitig die Nutzung dieses Angebots durch die Teilnehmenden beleuchtet (kompakt hierzu: Schrader et al. 2019, S. 778). Vergleichbar zu Kompetenzmodellen, die Lehr- oder Fachkräfte fokussieren, werden die Fortbildner*innen, ihr Wissen und Können in den Blick genommen, gleichzeitig müssen die Adressat*innen das vermittelte Wissen und Können als für ihre Praxis nützlich und anwendbar wahrnehmen (Lipowsky 2019), auch weitere Faktoren wie ihr Vorwissen, ihre Überzeugungen und Selbstwirksamkeit sind zentral (Lipowsky und Rzejak 2018). Ebenso ist der Kontext wie die wahrgenommene Unterstützung durch Leitung und Kollegium bedeutsam für den Erfolg von Fortbildungen. So werden verschiedene Komponenten der Wirksamkeit von Fortbildungen bestimmt (Lipowsky und Rzejak 2018).

Auch wenn die Modelle viele Faktoren zu berücksichtigen erlauben, ist weithin ungeklärt, wie sich der ,Transferprozess“ (Schrader et al. 2019, S. 778) in der Logik der Modelle konkret vollzieht bzw. wie das Zusammenspiel von „Disposition“, „Haltung“ und „Performanz“ (Fröhlich-Gildhoff et al. 2011, S. 17f.) theoretisch verstanden werden kann. Dieses Desiderat gilt insbesondere für Modelle, in denen nicht auf das psychologische Konstrukt der Überzeugungen als die einer Person bewusst zugängliche Werthaltung oder Einstellung rekurriert wird, sondern in denen mit dem Konzept der Haltung eine andere theoretische Bedeutung zugesprochen wird. Sie fungiert als ,handlungsgenerierende Struktur (...) ,hinter“ der Ebene der Disposition und beeinflusst wesentlich die Enaktierung von Dispositionen in die pädagogische Performanz" (Fröhlich-Gildhoff et al. 2011, S. 18; Hervorhebung der Autor*innen). Theoretisch ergiebig, um diese handlungsgenerierende Struktur konzeptionell zu fassen, sind handlungsleitende Orientierungen (Bohnsack 2010). Anders als das Konzept der Überzeugungen, das den kognitiven Merkmalen von Personen (Kunter und Pohlmann 2015, S. 264) zugeordnet wird und sowohl in Kompetenz- 
als auch in Angebots-Nutzungsmodellen breite Anwendung findet, verweisen Orientierungen auf habitualisierte Sinnmuster. Ihnen kommt eine zentrale „ScharnierFunktion“ zwischen Dispositionen und Handeln zu. Zugleich können diese nicht direkt verbalisiert, erfragt oder gemessen werden. Sie liegen dem Gesagten implizit zugrunde.

Diese Differenzen in der theoretischen Konzeptualisierung finden ihre Entsprechung in unterschiedlichen empirischen Zugangsweisen, die entweder eher eine quantitative, vielfach psychologisch orientierte Forschungslogik nahelegen oder aber stärker einer qualitativen Logik folgen und die Bedeutungs- und Sinnzuschreibungen des pädagogischen Personals in den Vordergrund rücken (u.a. Bischoff 2017; Viernickel et al. 2013; Vomhof 2016) und diese in Bezug auf Fortbildungen zu rekonstruieren erlauben.

\subsection{Forschungslage}

Der Forschungsstand umfasst Studien zu den Facetten professioneller (Sprachförder-)Kompetenzen (a) und zu den Wirkungen von Fortbildungen auch im Kontext Sprache bzw. Sprachförderung (b).

- (a) Verschiedene Studien zeigen auf, dass die Sprachförderkompetenzen von pädagogischen Fachkräften eine hohe Varianz aufweisen und insgesamt von eher niedrigerer Qualität sind (u. a. Faas 2013). Eine weitere Qualifizierung des pädagogischen Personals wird demzufolge für erforderlich erachtet. Allerdings konnten eindeutige Zusammenhänge zwischen dem bereichsspezifischen Wissen und Können und dem professionellen Handeln bislang nur vereinzelt nachgewiesen werden (Beckerle 2017; Ofner 2014); immer wieder werden sogenannte „Transferlücken“ zwischen den Kompetenzfacetten Wissen und Handeln festgestellt (Böhm et al. 2017; Faas 2013). Damit liegen noch keine ausreichend gesicherten empirischen Erkenntnisse darüber vor, in welchem Umfang Sprachförderkompetenzen des pädagogischen Personals notwendig sind und welche genau es sein sollen, damit erfolgreiche Sprachförderung gelingen kann (Kucharz 2018, S. 250). Dieses Desiderat gilt auch für den Übergang Kita-Grundschule (Erdogan et al. 2021) und für Facetten der Sprachförderkompetenz wie Motivation und Überzeugungen der Pädagog*innen sowie ihre handlungsleitenden Orientierungen, die bislang - im Kontext Sprache - kaum empirisch untersucht werden.

- (b) Generell gibt es eine hohe Fortbildungsbereitschaft bei Fachkräften in Kindertageseinrichtungen, die stärker ausgeprägt ist als bei anderen Berufsgruppen, u. a. Lehrkräften (Von Hippel 2011). Dabei teilen Fachkräfte selbst die Einschätzung eines Kompetenzdefizits nicht, wie es in der politischen und fachlichen Diskussion offenkundig wird (zu einer Übersicht: Betz 2013). Vielmehr weisen Studien darauf hin, dass Fachkräfte hohe Selbstwirksamkeitserfahrungen haben und ein insgesamt hohes Kompetenzerleben mit Blick auf die Bewältigung beruflicher Anforderungen (Beher und Walter 2012).

Ausgeprägt ist die Forschung zu Wirkungen von Fortbildungen v. a. in der Lehrer*innen- und Unterrichtsforschung (u.a. Lipowsky 2019; Lipowsky und Rzejak 2018; Übersichten für Kindertageseinrichtungen liefern Egert et al. 2018; Wirts 
et al. 2019.). Fortbildungen wird ein erhebliches Potenzial attestiert, „das Wissen und Handeln von Lehrpersonen zu beeinflussen und sich bis auf das Lernen von Schülern/-innen auszuwirken“ (Lipowsky und Rzejak 2018, S. 34). Als entscheidend gelten u.a. Kriterien des Angebots bzw. spezifischer Trainings wie aktivierende Methoden und (Video-)Feedback sowie die Intensität und der Umfang, wobei es in pädagogischen Handlungsfeldern keine einfachen linearen Zusammenhänge wie zwischen Fortbildungsangebot und -erfolg gibt (u.a. Egert et al. 2018).

Für eine Systematisierung der Forschungslandschaft wird im Folgenden eine Orientierung an der Unterscheidung der Wirkungsebenen von Kirkpatrick und Kirkpatrick (2006) vorgenommen, die knapp und berufsfeldübergreifend erfolgt. So zeigt sich in einer Übersicht über drei Ebenen, die auch für die empirischen Analysen wesentlich sind, Folgendes:

\subsubsection{Zufriedenheit und Akzeptanz}

Studien zeigen, dass Lehr- und Fachkräfte dann zufrieden sind, wenn die Fortbildung praxisnah gestaltet ist, Gelegenheit zum Austausch mit Kolleg*innen bietet und Fortbildner*innen kompetent sind. Lipowsky und Rzejak (2018) zeigen in einer Studienübersicht, dass hohe Zufriedenheit und Akzeptanz jedoch keine Zusammenhänge mit Wirkungen auf den anderen Ebenen haben müssen (S. 36). Wirts et al. (2019, S. 50) hingegen kommen für die Fortbildungsforschung zur frühen Kindheit zu dem Schluss, dass es einen nachweisbaren Zusammenhang ,zwischen Transfererfolg und einer höheren Zufriedenheit und Brauchbarkeitseinschätzung der Fortbildungsinhalte“" gibt.

Gerade im Kontext Sprachförderung zeigen Korrelationsanalysen zwischen der Bewertung der besuchten Fortbildung und Veränderungen in der Fachkraft-Kind-Interaktionsqualität ,,signifikant weniger negative Interaktionen [...] bei höherer Zufriedenheit/Spaß mit der [...] Fortbildung und höherer Einschätzung der Brauchbarkeit der FoBi-Inhalte, während sich signifikant schlechtere Lernarrangements im Zusammenhang mit einer höheren Einschätzung der Wissensvermittlung in der Fortbildung zeigten“" (Wirts et al. 2019, S. 49).

Zufriedenheit und Akzeptanz von Fortbildungen scheinen bedeutsam für den Transfererfolg zu sein, wenngleich die Zusammenhänge bisweilen uneindeutig sind oder sich nur teilweise erklären lassen.

\subsubsection{Lernerfolg bzw. Weiterentwicklung von Wissen und Können}

Mehrere Studien haben untersucht, welche Wirkungen Fortbildungen auf die Sprachförderkompetenz der Teilnehmenden haben (u. a. Armstrong et al. 2008; MarkussenBrown et al. 2017).

Im Entwicklungsprojekt TRIO (Schierbaum et al. 2020) z. B. wurden pädagogische Fachkräfte und Grundschullehrkräfte zwischen erstem und zweitem MZP in einer siebentägigen Fortbildungsreihe professionell über ein Dreivierteljahr hinweg geschult und in ihrem sprachlichen Arbeiten mit Kindern im letzten Kindergartenjahr gecoacht. Die Sprachförderkompetenz der Pädagog*innen wurde mit dem SprachKoPF (Thoma et al. 2014) erfasst. Hier zeigte sich, dass die Experimental- im 
Vergleich zur Kontrollgruppe ihre Kompetenzen signifikant verbessert hat, sowohl im Gesamtwert als auch im Bereich des Wissens; im Bereich des Könnens wird der Signifikanzwert 5\% nur knapp verfehlt. Das Evaluationsprojekt allE untersuchte die Sprachförderkompetenz von pädagogischen Fachkräften vergleichend für zwei Konzepte (Mackowiak et al. 2021): Konzept A enthielt zwischen den beiden MZP eine ausführliche Fortbildung zum Einsatz von Sprachfördertechniken, bei Konzept B gab es im Evaluationszeitraum keine zusätzlichen spezifischen Fortbildungen zur Sprachförderung über das übliche Angebot hinaus. Die Sprachförderkompetenz wurde u. a. anhand eines Vignettentests überprüft, der v. a. das diagnostische Wissen der Fachkräfte fokussierte. Hier zeigte sich, dass sich über die Zeit v. a. das Wissen der Fachkräfte von Konzept B steigerte, was auch schon zum 1. MZP höher ausfiel als das der Fachkräfte von Konzept A. Insgesamt gesehen lag das Niveau beider Gruppen allerdings relativ niedrig: von den in den Vignetten gezeigten Aspekten wurden kaum mehr als $10 \%$ genannt (Mackowiak et al. 2021).

Durch Fortbildungen lassen sich also Wirkungen auf das Wissen und Können des pädagogischen Personals nachweisen, allerdings nur in spezifischen Konstellationen und mit insgesamt geringen Effektstärken (vgl. die Metaanalysen internationaler Studien von Fukkink und Lont 2007; Markussen-Brown et al. 2017). Auch zeigen Studien zu Lehrkraft-Schüler*innen- bzw. Fachkraft-Kind-Interaktionen die Veränderbarkeit von Überzeugungen durch Fortbildungen im Kontext Sprachförderung auf (u. a. Hamre et al. 2012). Handlungsleitende Orientierungen der Pädagog*innen, die Aufschlüsse über den modus operandi der Fachkräfte geben, werden in den überwiegend quantitativ ausgerichteten Studien nicht untersucht; sie werden in MetaAnalysen im Kontext Sprache nicht berücksichtigt.

\subsubsection{Transfer, Handeln bzw. Performanz}

Der direkte Nachweis eines veränderten Handelns nach dem Besuch von Fortbildungen gestaltet sich schwierig, die Zusammenhänge sind komplex. Auch im Kontext Sprachförderung (vgl. die Metaanalyse von Markussen-Brown et al. 2017) zeigen die Befunde z. B. aus BiSS-Evaluationsstudien, dass trotz Fortbildungen nur geringe Veränderungen in der Praxis beobachtbar waren. So reduzierte sich, basierend auf Feinanalysen von Videos aus Bilderbuchbetrachtungen, der Redeanteil der Fachkräfte und der Redeanteil der Kinder stieg an. Diese Beobachtung ließ sich allerdings für Freispielsituationen nicht replizieren (Wirts et al. 2019, S. 73). Ebenfalls ließen sich keine Unterschiede vor und nach der Fortbildung nachweisen beim Einsatz sprachförderlicher Interaktionsstrategien oder der Häufigkeit sprachlicher Bildungsaktivitäten (Wirts et al. 2019). Nachweisbare Verbesserungen gab es in mehreren Bereichen der Interaktionsqualität bei den Teilnehmenden, die eine längere Videoberatung erhalten haben. Im Projekt allE konnte dagegen eine deutliche Steigerung im Einsatz von Sprachfördertechniken nachgewiesen werden bei der Gruppe, die im Verlauf der Evaluation eine ausführliche Fortbildungsreihe besucht hatte (Mackowiak et al. 2021). Weitere solcher Studienbefunde finden sich bei Kammermeyer et al. (2019) sowie Simon und Sachse (2011), die im Gegensatz zu den erwähnten BiSSStudien über ein experimentelles randomisiertes Kontrollgruppen-Design verfügen, so dass deren Ergebnisse belastbarer sind. 
Quer zu diesen Ebenen liegen die je gewählten empirischen Zugänge zur Erforschung der Wirksamkeit von Fortbildungen. Gerade die empirische Erfassung der Überzeugungen sowie des Wissens und Könnens des pädagogischen Personals ist divers. Zum Einsatz kommen u.a. die Überprüfung des Wissens auf der Basis spezifischer Tests (u.a. SprachKoPF), die Selbsteinschätzungen der Befragten in Bezug auf Kenntnisse und Fähigkeiten, das Einholen von Beurteilungen anderer (z.B. Schüler*innenbefragungen), die direkte Beobachtung z. B. über Videografie (Erdogan et al. 2021) oder Beobachtungsskalen. Trotz der Vielfalt der Zugänge ist beobachtbar, dass die große Mehrheit quantitativ-empirisch die Zusammenhänge und Wirkungen bestimmt; qualitative Wirkungsforschung ist weitaus weniger verbreitet (u. a. Cloos et al. 2013), im Kontext Sprachförderung quasi nicht existent.

Demnach bleibt zusammenfassend festzuhalten: Wirkungen auf das sprachförderliche Handeln konnte bislang in Studien nur dann eindeutig gezeigt werden, wenn die Pädagog*innen im Verlauf der Evaluation umfangreiche, breit angelegte und praxisnahe Fortbildungen zur Sprachförderung besucht hatten (u. a. Beckerle 2017; Kammermeyer et al. 2019; Schierbaum et al. 2020; Simon und Sachse 2011); für unspezifische oder vereinzelte Fortbildungen waren die Befunde uneindeutig (z.B. Erdogan et al. 2021; Mackowiak et al. 2021; Wirts et al. 2019). Des Weiteren muss festgehalten werden, dass die Kompetenzmodelle zwar der ,professionellen Haltung“ (Schrader et al. 2019, S. 781) eine große Bedeutung zusprechen, dieses Konstrukt oder das der handlungsleitenden Orientierungen aber in empirischen Studien zur Sprachförderung nicht untersucht wurden. Gleichzeitig werden Akzeptanz und wahrgenommene Bedeutung für die eigene Praxis als wichtige Voraussetzungen angesehen, damit Fortbildungen Wirkung entfalten können.

\subsection{Entwicklung der Fragestellungen}

Entsprechend der Forschungsdesiderate wird im Folgenden mithilfe quantitativer und v. a. qualitativer Zugänge das Zusammenspiel zwischen Sprachförderkompetenzen, Fortbildungsbesuch und handlungsleitenden Orientierungen von Pädagog*innen in Bezug auf Sprachförderung am Übergang Kita-Grundschule untersucht. Hierfür wird auf eine im Projekt SPRÜNGE (Sprachförderung im Übergang Kindergarten Grundschule evaluieren) empirisch erarbeitete Typenbildung (Schmidt-Hertha und Tippelt 2011) zurückgegriffen, die ,,als eine Strategie der Informationsreduktion bei gleichzeitigem Wissensgewinn“ (S. 23) dazu dient, explorative Einblicke in die Wirkungen von Fortbildungen für verschiedene Sprachfördertypen zu erhalten. Folgende Teilfragestellungen werden verfolgt:

1. Wie unterscheiden sich Fach- und Lehrkräfte vor dem Hintergrund ihrer handlungsleitenden Orientierungen in ihren Sprachförderkompetenzen und im Fortbildungsbesuch?

2. Wie unterscheiden sich die selbstberichteten Wirkungen der Fortbildungen? 


\section{Forschungsdesign und methodisches Vorgehen}

Die Daten stammen aus der multiperspektivischen, längsschnittlichen Evaluationsstudie SPRÜNGE, die im Rahmen von BiSS unterschiedliche Formen der Sprachförderung (Lernwerkstätten, Projektarbeit, Vorkurse), der Fortbildung und Kooperation am Übergang Kita-Grundschule zu drei Messzeitpunkten (MZP) ${ }^{2}$ im Hinblick auf ihre Wirksamkeit mit quantitativen und qualitativen methodischen Zugängen untersucht hat.

Zur Beantwortung der ersten Frage gingen Daten aus einer Teilstichprobe von 17 Fach- und 10 Lehrkräften aus dem Projekt ein. Es wurden alle diejenigen ausgewählt, von denen Daten zu mindestens zwei MZP (prä und post) sowohl vom Online-Test SprachKoPF als auch aus qualitativen Interviews vorlagen. Für Fragestellung zwei wurde davon eine Teilgruppe fokussiert $(n=10)$, die besonders in die Sprachförderarbeit involviert war; alle Teilnehmenden sind weiblich, nur für eine Fachkraft ist Deutsch nicht die Erstsprache. ${ }^{3}$

\subsection{Anwendungsbezogenes Wissen und Können}

Die Sprachförderkompetenzen der Fach- und Lehrkräfte wurden mittels des Online-

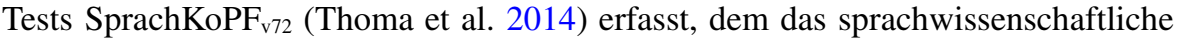
Sprachförderkompetenzmodell von Hopp et al. (2010) zugrunde liegt. Für die erste Frage werden die Skalen zum anwendungsbezogenen Wissen mit 33 Items sowie zum Können mit 18 Items herangezogen. Die Items zum anwendungsbezogenen Wissen betreffen Themen wie Diagnostik und Spracherwerb; hier geht es v.a. um das Erkennen von Meilensteinen bei jungen Kindern (2-4 Jahre), um die Anwendung von Erhebungsverfahren sowie darum, was man mit bestimmten Fördermaterialien genau fördern kann. Im Bereich des Könnens werden Handlungsoptionen anhand konkreter Sprachfördersituationen erfragt. Konkrete Situationen in Kindertageseinrichtungen oder Handlungen von Fachkräften sollen bzgl. ihres sprachförderlichen Potentials sowie Äußerungen von Kindern diagnostisch eingeschätzt und passende Dokumentationen benannt werden. Einschränkend ist zu erwähnen, dass die Szenen aus Kitas stammen, so dass sie für die Lehrkräfte nicht unbedingt passgenau sind.

Da sich die Kompetenzen im anwendungsbezogenen Wissen und im Können über die Evaluationszeit hinweg nicht bedeutsam verändert haben (Erdogan et al. 2021), wird für die Analysen lediglich der Post-MZP herangezogen.

\footnotetext{
2 Es wurden eine Kinderkohorte von Anfang des letzten Kita-Jahres bis Ende des ersten Schuljahres sowie die an der Sprachförderung beteiligten Pädagog*innen begleitet. Die Erhebungen lagen im Abstand von jeweils knapp einem Jahr (Sommer 2016-Sommer 2018).

3 Der hohe Dropout in der Stichprobe ist auf die insgesamt große Skepsis der beteiligten Verbünde gegenüber der Evaluation zurückzuführen. Einige Verbünde verweigerten ihre Teilnahme daran ganz, in anderen lehnten v. a. die Lehrkräfte eine Teilnahme ab. Insbesondere stieß der Online-Test SprachKoPF an sich auf große Ablehnung (ca. Zweidrittel). Inwieweit dadurch eine Verzerrung der Stichprobe vorliegt, kann nicht eindeutig geklärt werden. Es fehlen dadurch v. a. weitere Lehr- und Fachkräfte mit mehrsprachigem Hintergrund. Dass nur besonders engagierte und kompetente Pädagog*innen teilgenommen haben, kann nicht bestätigt werden, denn die Testwerte zeigen eine große Bandbreite, eventuell trifft dies für die Lehrkräfte $\mathrm{zu}$.
} 


\subsection{Hintergrundvariablen}

Folgende im SprachKoPF erfasste Hintergrundvariablen werden herangezogen: Berufsgruppe Fach- oder Lehrkraft, Berufserfahrung in Jahren und die Dauer der besuchten Fortbildungen in Stunden. In einer Multiple-Choice-Liste wurde angekreuzt, was man in welchen der genannten elf Sprachförderbereiche (s. Tab. 4) in den Fortbildungen dazu gelernt hat.

\subsection{Handlungsleitende Orientierungen: Sprachfördertypen}

$\mathrm{Zu}$ den jeweiligen MZP wurden leitfadengestützte Interviews mit den Fach- und Lehrkräften durchgeführt. Die Interviews zielten u. a. darauf ab, ihre Perspektiven zu Sprachförderung am Übergang und zu Wirkungsweisen der Fortbildungen zu erfassen, um damit einerseits Einblicke in ihre Motivation zu erhalten und andererseits ihre handlungsleitenden Orientierungen in Bezug auf Sprachförderung rekonstruieren zu können. Zum Thema Fortbildung wurde u. a. erfragt, wie es zur Teilnahme an den Fortbildungen kam, welche Rolle die Inhalte für die praktische Arbeit spielen und wie beurteilt wird, dass Fortbildungen von Fach- und Lehrkräften gemeinsam besucht werden.

Die dem Sprachförderhandeln zugrundeliegenden handlungsleitenden Orientierungen wurden fallbezogen mittels Dokumentarischer Methode rekonstruiert. Dabei wurde nicht nur das, was (in Interviews) gesagt wurde, analysiert, sondern auch rekonstruiert, wie es gesagt wurde (weiterführend: Bohnsack 2010). Damit wurden systematisch differenzierbare Bearbeitungs- und Bewältigungsweisen dazu deutlich, wie die Beteiligten mit der Anforderung umgehen, die deutsche Sprache am Übergang zu fördern. Im Ergebnis der fallinternen und -externen Kontrastierung konnten vier sinngenetische Typen identifiziert werden (ausführlich Kämpfe und Betz 2020; Überblick zur Methode: Schmidt-Hertha und Tippelt 2011, S. 28 f.; Viernickel et al. 2013).

Die Typen variieren entlang der Differenzlinie der strukturellen Involviertheit in Sprachförderung am Übergang. Als weitere Differenzlinie in den Orientierungen lässt sich eine Kita- bzw. Schulzentrierung in der Sprachförderung ausmachen. Für den intuitiv-schematischen (Typ 1) und den didaktischen Sprachfördertyp (Typ 2) ist eine Kitazentrierung, für den versierten (Typ 3) und den konzeptionell-planvollen Sprachfördertyp (Typ 4) ist eine Schulzentrierung charakteristisch. Die Handlungsorientierungen spiegeln die strukturellen Vorgaben der jeweiligen Berufspraxis und -gruppe: in der Kita ein eher freier und offener Umgang mit Sprachförderung, der stärker auf die Belange der Kinder ausgerichtet ist, während auf der anderen Seite die Anforderungen der Schule und ein planvolles Vorgehen das Handeln rahmen. Die Typen unterscheiden sich also nach Berufsgruppenzugehörigkeit. Eine geringere Involviertheit (Typ $1 \& 4$ ) in die Sprachförderung geht mit einem ökonomischen, auf bewährten Mustern basierendem und dabei eher schematischen bzw. standardisierten Vorgehen einher, während eine höhere Involviertheit (Typ 2 \& 3) auf ein an den konkreten Bedürfnissen und Rahmenbedingungen orientiertes adaptives Vorgehen hinweist, verbunden mit dem Anspruch stetiger Optimierung. 
Tab. 1 Stichprobenbeschreibung, differenziert nach Sprachfördertypen

\begin{tabular}{lllll}
\hline Typ & $\begin{array}{l}\text { Typ 1: intuitiv- } \\
\text { schematischer } \\
\text { Typ }\end{array}$ & $\begin{array}{l}\text { Typ 2: didakti- } \\
\text { scher Typ }\end{array}$ & $\begin{array}{l}\text { Typ 3: versierter } \\
\text { Typ }\end{array}$ & $\begin{array}{l}\text { Typ 4: konzep- } \\
\text { tionell-planvoller } \\
\text { Typ }\end{array}$ \\
\hline Anzahl & $n=12$ & $n=5$ & $n=5$ & $n=5$ \\
Berufsgruppe & Fachkraft & Fachkraft & Lehrkraft & Lehrkraft \\
$\begin{array}{l}\text { Durchschnittl. } \\
\text { Berufserfahrung }\end{array}$ & 11,4 Jahre (min & 19,2 Jahre (min & 11,6 Jahre (min & 16,25 Jahre (min \\
\hline
\end{tabular}

${ }^{a}$ Es gibt keinen signifikanten Unterschied in der Berufserfahrung zwischen den vier Typen

Die Analysen (4.2.) legen mit dem Fokus auf Fortbildungen einen themenbezogenen Vergleichshorizont an die rekonstruierten Typen an, d.h. es wird untersucht, inwiefern sich die (Sprachförder-)Typen mit Blick auf (gemeinsam) besuchte Fortbildungen unterscheiden.

\subsection{Stichprobenbeschreibung}

Tabelle 1 zeigt die Verteilung der 17 Fach- und 10 Lehrkräfte auf die oben beschriebenen vier Typen.

Alle Analysen in 4.1 beziehen sich zunächst auf diese Stichprobe, sodann in 4.2 auf die Fach- und Lehrkräfte der beiden Typen 2 und 3.

\section{Ergebnisse}

\subsection{Wie unterscheiden sich Fach- und Lehrkräfte vor dem Hintergrund ihrer handlungsleitenden Orientierungen in ihren Sprachförderkompetenzen und im Fortbildungsbesuch?}

\subsubsection{Sprachförderkompetenzen}

Tab. 2 zeigt, dass die Lehrkräfte über signifikant höhere Werte im anwendungsbezogenen Wissen verfügen als die Fachkräfte $(p<0,05)$; der Unterschied im Können ist dagegen nicht bedeutsam $(p>0,05)$.

Vergleicht man die beiden Berufsgruppen hinsichtlich der durchschnittlichen Dauer ihrer besuchten Fortbildungen, zeigen sich zwar deskriptive, aber keine signifikanten Unterschiede, was auf die große Streuung zurückzuführen ist (Fk: MW=73,6 h (SD 168,0); Lk: MW=167,5 h (SD 312,6)).

Tab. 2 Vergleich der Mittelwerte im Sprachkopf zum praxisbezogenen Wissen und Können zwischen Fach- (Fk) und Lehrkräften (Lk)

\begin{tabular}{lllll}
\hline & Fk $(n=17)$ & Lk $(n=10)$ & (df) t-Wert & $p$-Wert \\
\hline Anwendungsbez. Wissen & $0,46($ SD 0,18) & 0,64 (SD 0,07) & $(25)-2,85$ & 0,009 \\
Können & 0,27 (SD 0,14) & $0,37($ SD 0,12) & $(25)-1,74$ & 0,09 \\
\hline
\end{tabular}

T-test für unabhängige Stichproben. Testwerte zwischen 0 und 1 
Tab. 3 Anzahl der durchschnittlich besuchten Fortbildungsstunden und Mittelwerte im anwendungsbezogenen Wissen und Können differenziert nach den vier Sprachfördertypen

\begin{tabular}{lllll}
\hline & Typ 1 (Fk $n=12)$ & Typ 2 (Fk $n=5)$ & Typ 3 (Lk $n=5)$ & Typ 4 (Lk $n=5)$ \\
\hline Fortbildungsstunden & $5,75 \mathrm{~h}$ & $217,2 \mathrm{~h}$ & $259,8 \mathrm{~h}$ & $75,2 \mathrm{~h}$ \\
Anwendungsbez. & $0,37(\mathrm{SD} 0,09)$ & $0,55(\mathrm{SD} 0,09)$ & $0,65(\mathrm{SD} 0,11)$ & $0,61(\mathrm{SD} 0,07)$ \\
Wissen & & & & \\
Können & $0,19(\mathrm{SD} \mathrm{0,14)}$ & $0,39(\mathrm{SD} 0,16)$ & $0,45(\mathrm{SD} 0,15)$ & $0,33(\mathrm{SD} 0,13)$ \\
\hline
\end{tabular}

Dauer in Stunden aller besuchten Fortbildungen zur Sprachförderung. Testwerte zwischen 0 und 1

Da die Werte insgesamt eine sehr große Streuung aufweisen, die die Heterogenität des Feldes widerspiegeln, sollen für den weiteren Erkenntnisgewinn die Vergleiche für die vier Sprachfördertypen ausdifferenziert werden, um sie für weitere deskriptive und qualitative Analysen zugänglich zu machen (vgl. Tab. 3).

Aus Tab. 3 wird ersichtlich, dass sich Typ 1, der intuitiv-schematische Typ, deutlich von den anderen Typen unterscheidet: es wurden insgesamt nur sehr wenige Fortbildungen besucht und die Werte im Sprachtest liegen deutlich unter denen der anderen Typen. Die non-parametrische paarweise Testung (Kruskall-Wallis Test) ergibt keine signifikanten Unterschiede für das Können, aber für das anwendungsbezogene Wissen $(p \leq 0,005)$. Die anderen Typen unterscheiden sich nicht deutlich voneinander.

Tab. 4 Angaben, in welchem Bereich durch die Fortbildungen vor allem dazu gelernt wurde (Anzahl der Nennungen, Mehrfachnennungen)

\begin{tabular}{|c|c|c|c|c|c|}
\hline $\begin{array}{l}\text { Themenbereiche } \\
\text { (s. Kap 2.1) }\end{array}$ & $\begin{array}{l}\text { Sprachbereich (entspricht Mul- } \\
\text { tiple-Choice-Liste im Sprach- } \\
\text { KoPF) }\end{array}$ & $\begin{array}{l}\text { Typ } 1 \\
(12 \mathrm{Fk})\end{array}$ & $\begin{array}{l}\text { Typ } 2 \\
(5 \mathrm{Fk})\end{array}$ & $\begin{array}{l}\text { Typ } 3 \\
(5 \mathrm{Lk})\end{array}$ & $\begin{array}{l}\text { Typ } 4 \\
(5 \mathrm{Lk})\end{array}$ \\
\hline \multirow{3}{*}{$\begin{array}{l}\text { Grundlagen der } \\
\text { deutschen } \\
\text { Sprache }\end{array}$} & Phonologie & 1 & 5 & 3 & 0 \\
\hline & $\begin{array}{l}\text { Sprachliche Strukturen (z. B. } \\
\text { Grammatik) }\end{array}$ & 4 & 5 & 8 & 2 \\
\hline & Wortschatz & 3 & 5 & 7 & 1 \\
\hline \multirow{2}{*}{$\begin{array}{l}\text { Erst- und } \\
\text { Zweitspracher- } \\
\text { werb und } \\
\text { Mehrsprachig- } \\
\text { keit }\end{array}$} & $\begin{array}{l}\text { Spracherwerb \& Sprachent- } \\
\text { wicklung }\end{array}$ & 1 & 5 & 5 & 1 \\
\hline & Umgang mit Multikulturalität & 2 & 4 & 7 & 2 \\
\hline \multirow{2}{*}{$\begin{array}{l}\text { Planung und } \\
\text { Durchführung }\end{array}$} & Sprachförderung & 3 & 5 & 6 & 3 \\
\hline & Reime und Lieder & 1 & 7 & 1 & 1 \\
\hline Diagnostik & Sprachdiagnostik & 2 & 3 & 4 & 1 \\
\hline Literacy & $\begin{array}{l}\text { Literacy (Lese- und Schreiber- } \\
\text { werb) }\end{array}$ & 3 & 6 & 6 & 2 \\
\hline Elternarbeit & Kommunikation mit Eltern & 0 & 5 & 2 & 3 \\
\hline $\begin{array}{l}\text { Nicht bereichs- } \\
\text { spezifisch }\end{array}$ & Selbstmanagement & 0 & 5 & 2 & 1 \\
\hline \multicolumn{2}{|c|}{$\begin{array}{l}\text { Durchschnittl. Anzahl der Nennungen pro Per- } \\
\text { son }\end{array}$} & 1,6 & 11 & 10,2 & 3,4 \\
\hline
\end{tabular}




\subsubsection{Fortbildungsbesuch}

Typ 2 und 3 weisen einen deutlich umfangreicheren Fortbildungsbesuch auf als die anderen beiden Sprachfördertypen. Drei Fachkräfte des Typ 2 und zwei Lehrkräfte des Typ 3 haben bereits vor dem Evaluationszeitraum eine mehrtägige Weiterbildung von mehr als $100 \mathrm{~h}$ Dauer zur Sprachförderkraft absolviert.

Tab. 4 zeigt die Einschätzungen der Fach- und Lehrkräfte dazu, in welchen Sprachbereichen sie in den Fortbildungen dazugelernt haben. Die Angaben für die Sprachfördertypen sind getrennt aufgeführt, es handelt sich um die addierte Anzahl an Nennungen.

Die Tabelle zeigt, dass alle vier Sprachfördertypen mehrere Inhalte, die in der Liste im SprachKoPF zur Auswahl standen, angegeben haben. Insbesondere Typ 2 und 3 gaben mit durchschnittlich elf Nennungen pro Person an, dass sie in vielen Wissensbereichen mehrfach dazu gelernt haben und damit ihrer Einschätzung nach über breite Wissens- und Könnens-Kompetenzen verfügen. Wesentliche Unterschiede finden sich lediglich bei dem Thema „Reime und Lieder“, was typischerweise eher im Kitabereich Bedeutung hat.

Zusammenfassend kann festgestellt werden, dass sich die große Heterogenität in den Sprachförderkompetenzen von Pädagog*innen v. a. auf eine Gruppe zurückführen lässt, die im Rahmen des Projektes als intuitiv-schematischer Sprachfördertyp identifiziert wurde. Die Fachkräfte dieses Typs verfügen über niedrige Testwerte, haben nur wenige Fortbildungen besucht und identifizieren sich nur wenig mit Sprachförderung. Gleichzeitig machen sie in der Stichprobe die größte Gruppe aus. Auch die Lehrkräfte von Typ 4 haben vergleichsweise wenige Fortbildungen besucht, schneiden aber in den Testwerten deutlich besser ab. Typ 2 und 3 verbindet ein besonderes Engagement in der Sprachförderung, das sich auch deutlich hinsichtlich ihrer Kompetenzwerte und ihres Fortbildungsbesuchs zeigt. Um diese beiden Typen weiter explorieren zu können, ihre Deutungsmuster in Bezug auf die Wirkungen von Fortbildungen zu verstehen, werden im Folgenden ihre handlungsleitenden Orientierungen und damit ihr habitualisiertes und implizites Wissen genauer betrachtet.

\subsection{Wie unterscheiden sich die selbstberichteten Wirkungen der Fortbildungen?}

Im Folgenden werden die Perspektiven des didaktischen und des versierten Sprachfördertyps miteinander kontrastiert. Zwar vertritt jeder Typ eine der beiden Berufsgruppen, dennoch verbindet sie ihre umfangreichen Fortbildungen, ihre verhältnismäßig hohen Testwerte im SprachKoPF sowie ihre große Involviertheit in die Sprachförderung. Über den qualitativ-rekonstruktiven Zugang aus den Interviews kann die über den SprachKoPF vermittelte Homogenität der beiden Typen näher ergründet und weiter ausdifferenziert werden. Die Darstellung der Ergebnisse erfolgt in Anlehnung an die drei Ebenen des Fortbildungserfolgs von Kirkpatrick und Kirkpatrick (2006; vgl. Kap. 2.2). Die auf den Ebenen identifizierten Kriterien werden jeweils nach Typen differenziert sowie mit Blick auf Gemeinsamkeiten nachvollzogen. 


\subsubsection{Zufriedenheit und Akzeptanz}

Zufriedenheit und Akzeptanz in Bezug auf sprachförderbezogene (BiSS-)Fortbildungen werden typenübergreifend - analog zu anderen Studien - insbesondere an eine hohe Praxisrelevanz der Fortbildungsinhalte sowie an die Effizienz der Fortbildungsformate geknüpft. Beide Kriterien werden jedoch von den Typen unterschiedlich ausgedeutet.

Der didaktische Typ orientiert sich bei der Beurteilung von Praxisrelevanz an kindzentrierten und situationsorientierten Sprachförderzugängen. Als zentral für diese adaptive Vorgehensweise (,immer orientiert an den Bedürfnissen der Kinder" $\left.\left(\mathrm{T}_{2} 2{ }_{2}{ }^{4}\right)^{4}\right)$ erweist sich die Strategie einer impliziten Förderung (,ohne dass sie überhaupt merken, dass sie jetzt was machen, was die anderen nicht machen müssen." (T2_3_1) ). Besonderes Augenmerk wird auch auf die Art und Weise gelegt, wie Alltagssituationen versprachlicht werden. Als besonders praxisrelevant und damit ertragreich gelten Inhalte und Formate, denen ein in Bezug auf die Sprachförderung allgemein aktivierender Charakter zugeschrieben wird, d.h., dass die Fortbildungen die Fachkräfte unterstützen, um Kinder ganz allgemein zum Sprechen und Erzählen zu motivieren. Genannt werden unterschiedliche ,aktivierende“ Sprachlehrstrategien (z.B. dialogisches Lesen, Scaffolding, Präsentationstechniken), -maßnahmen (z. B. Lernszenarien, entdeckendes Lernen, kreatives Gestalten, Lieder) und -medien (z.B. Bücher, Erzählkasten, Handpuppen). Thematisiert werden eher die didaktischen Zugänge als deren spezifisches Förderziel, und diese auch mit Blick auf deren ganzheitlichen Nutzen bewertet: „Also von daher sind so ne multifunktionalen Sprachförderangebote, die man [...] nicht nur zur Sprachförderung, sondern im Alltag integriert, im gesamten Kontext nutzen kann, optimal." (T2_2_1).

Vom versierten Typ werden demgegenüber insbesondere Fortbildungsinhalte und -formate dann als für die Praxis relevant eingeschätzt, wenn sie spezifische Förderbereiche oder Sprachdiagnostik mit Blick auf ein zu erreichendes Outcome ansprechen.

Da haben wir so [themenspezifische] Klassenkisten entwickelt, wo die Kinder über diese sachliche Grundlage dann tatsächlich an Sprache arbeiten, also an Satzbau, funktioniert die Wortschatzerweiterung und Präsentation von Arbeitsergebnissen. (T3_1_3).

Neben sprachlichen Strukturen, Wortschatzerweiterung, Präsentation von Arbeitsergebnissen und der Verknüpfung von Sachthemen mit Sprachbildung werden u.a. Phonologie und strukturiertes mündliches Erzählen als wichtige Grundlagen für den Lese- und Schreiberwerb, allgemein ,richtig sprechen“ ${ }_{\left({ }_{(3}{ }_{-} 5_{-3}\right)}$, der Ausbau der Bildungssprache sowie Sprachstandserhebungsverfahren genannt. Bei einem durch die Fortbildung begleiteten Aufbau einer (vorschulischen) Lernwerkstatt beispielsweise wird zudem die schulische Sozialisation als bedeutsames Lernziel hervorgehoben und in Kontrast zu anderen Lernwerkstätten im Verbund gesetzt:

[...] dass die Kinder [...] also schon herangeführt werden, was ist ein Arbeitsplan und selbstständig sich zu organisieren und einzuschätzen, also die haben

\footnotetext{
${ }^{4}$ Die Kürzel bilden Typ (T2/3), Person (Fach- bzw. Lehrkraft 1-5) und MZP ab (1/3).
} 
bestimmte Pflichtaufgaben die Kinder, also recht einfach gestaltet noch [...] Und das wurde von anderen Einrichtungen so, oh, das ist viel zu verschult, aber wir sehen jetzt, dass das für die Kinder machbar ist. [...]. (T3_5_1).

Dagegen werden vorgestellte Methoden wie die dialogische Bilderbuchbetrachtung zwar als interessanter Input (,,im Kindergarten ist es auf alle Fälle sinnvoll und ganz ne nette Sache"), aber für die eigene Umsetzung in einem Vorkurs als nicht ,zielgerichtet“ genug zurückgewiesen: ,viel zu viel durcheinander““ ${ }_{\left({ }_{3}{ }_{-} 4_{1}\right) \text {. }}$

Nach Ansicht beider Typen zeigen die lokalen BiSS-Veranstaltungen ein hohes Potenzial an Praxisrelevanz, besonders dann, wenn sie - wie in Inhouse-Fortbildungen - genau auf die Teilnehmenden ,zugeschnitten“ ${ }_{\left({ }_{(3}{ }_{-1} 1_{1}\right)}$ sind. Die Fortbildungsinhalte werden dabei überwiegend als (unverbindliche) Angebote (,Input") für die eigene Sprachförderpraxis verstanden, aus deren Fundus frei gewählt werden kann. Das besondere Format der von Fach- und Lehrkräften gemeinsam besuchten Fortbildungen zeigt, dass die divergierenden Handlungsorientierungen der Teilnehmenden bei der Beurteilung der Praxisrelevanz mitunter kollidieren und dabei Fortbildungsinhalte entlang der Differenzlinie von Kita- und Schulzentrierung für sich selbst als nicht kompatibel eingeschätzt werden: ,oft sehr schulstrukturiert und nicht so sehr Kita. “ (Т2___3); , Portfolio betrifft mich ja jetzt so gar nicht (.) also es ist manchmal vom Thema her jetzt schon sehr kindergartenspezifisch" (T3_4_1).

Ein weiteres wichtiges Kriterium zur typenübergreifenden Beurteilung gelungener Fortbildungen stellt der Grad an wahrgenommener Effizienz dar. Zum einen soll die Fortbildungszeit, die als kostbar, da knappes Gut bezeichnet wird, selbst effizient gestaltet sein, zum anderen sollen die praxisrelevanten Impulse unmittelbar anwendbar und mit den jeweiligen vorgefundenen (strukturellen) Bedingungen vereinbar sein. Während der didaktische Typ - zwar an bestimmte Ressourcen gebunden - hier einen freieren Handlungsspielraum beschreibt, sieht sich der versierte Typ stärker durch die Bedingung, ,Rahmenplan-konform““ ${ }_{(\text {T3____3) }}$ Zu agieren, beschränkt.

Die beiden bereits umfangreich fortgebildeten Typen verbindet im Zeitverlauf zudem ein gewisses Sättigungsempfinden an, wenn auch praxisnahen, Fortbildungsinhalten und Austauschtreffen:

im Endeffekt dann [zuletzt nur noch] Projekte vorgestellt wurden, wo die Ideen aus dem Programm stammten. Und ich muss nicht zugucken, wie eine andere Einrichtung Märchen erzählt. (T2_3_3).

Mich wundert's jetzt, dass es verlängert wurde, [...] und was da jetzt noch kommen kann und soll. [...] Wir wissen längst, was wir zu tun haben und wie's geht.

(T3_4_3).

\subsubsection{Lernerfolg bzw. Weiterentwicklung von Wissen und Können}

Die selbstberichteten Lernerfolge reichen bei beiden Typen von punktuellen Erkenntnissen bis hin zu umfänglichen Erweiterungen des eigenen Wissens und Könnens. Als zentralen Lernerfolg beschreiben sie, dass durch die Fortbildungen eine allgemein höhere Sensibilität geschaffen und ein Reflexionsprozess über das eigene sprachförderliche Handeln und Sprechen in Gang gesetzt wurden. Durch offenes 
Fragen ein offeneres Denken und Sprechen anzuregen wird als ,was so ganz Exis-

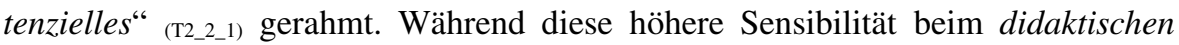
Typ, wie das folgende erste Zitat zeigt, vermehrt mit einem Reflexionsprozess in der Rolle als Sprachvorbild und dem Ziel selbst ,gut zu sprechen“ verbunden ist, reflektiert der versierte Typ Strategien ,richtiger Fragen' wie im zweiten Zitat vor dem Hintergrund schultypisch bislang weniger dialogisch ausgerichteter LehrkraftSchüler*innen-Interaktion: , viel über sich selber gelernt ((lacht)), [...] wie redet man mit den Kindern. “ (T2_2_3) ,, wie frag ich eigentlich, dass es auch offen gestaltet ist und nicht dieses typische alte Schulalltag Frage-Antwort-Spiel. “(13_2_3).

Geknüpft ist diese erhöhte Sensibilität an die typenübergreifend formulierte Erkenntnis, - unter Hilfestellung von entsprechenden Sprachlehrstrategien - Kinder in der sprachlichen Förderung vor Herausforderungen zu stellen (,nicht die Sprache zu

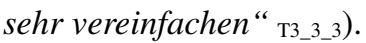

Zudem zeigen sich beide Typen für durchgängige Sprachbildung sensibilisiert; sie ordnen sich mit ihrer Sprachförderung am Übergang darin ein (,Die Wichtigkeit der Sprachentwicklung wird natürlich nochmal ganz doll hervorgehoben, auch für

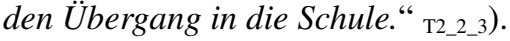

Die konkrete Erweiterung des eigenen Wissens und Könnens wird vom didaktischen Typ eher unspezifisch, vage beschrieben mit Verweisen auf konkrete und als attraktiv erachtete Materialien und Methoden (s. oben). Wie das erworbene Wissen und Können in didaktische Zugänge mündet, bleibt jedoch eher implizit.

Der versierte Typ knüpft seinen Zugewinn an Wissen und Können hingegen explizit an seine spezifischen Fokusse in der Sprachförderung an:

Also ich hab z. B., ja, mit rausgenommen, wie man eben mündliches Erzählen so strukturieren kann. [...], dass man auch nicht die Sprache zu sehr vereinfachen darf, sondern dass es auch unsere Aufgabe ist, die Kinder wirklich zur Bildungssprache hinzuführen, Ich hab gelernt, dass man Texte vorentlasten soll, indem man eben so einen kleinen [...] Advanced Organiser gibt oder dass man eben auch Texte [...] durch Fragen vorstrukturiert. Ich hab gelernt, dass man eben durch Scaffolding, indem man eben Satzanfänge vorgibt, auch das Sprechen erleichtern kann. Ja, ich hab gelernt, wie man ein Lernszenario umsetzt.

(T3_3_3).

\subsubsection{Handeln und Performanz}

Auf der Ebene der Interaktion wird über den Einsatz von Methoden und Material berichtet, die analog zu den jeweils präferierten Handlungsansätzen in die Sprachförderarbeit in Folge der Fortbildung übernommen wurden. Eine Sprachlehrstrategie, die von beiden Typen besonders häufig benannt wird, ist das Scaffolding, das die Kinder durch Formulierungshilfen bei dem Ziel ein offeneres Denken und Sprechen zu erzeugen unterstützt und für den jeweiligen Bildungsbereich adaptiert wird. Hervorgehoben wird neben der Vielfalt und Einfachheit an Einsatzmöglichkeiten auch die typenübergreifend wahrgenommene Resonanz bei den Kindern (,Und wir haben gemerkt, das funktioniert wirklich, ((lacht)) also die Kinder mussten immer 
sagen, mit wem habt ihr heute gearbeitet, oder habt ihr alleine gearbeitet, was habt ihr gemacht, [...]. “"(T2_1_3)).

Ein zentraler Unterschied zeichnet sich darin ab, nach welchen Kriterien die eigene Umsetzung der unterschiedlichen Methoden und Materialien evaluiert wird. Der didaktische Typ markiert das Befinden der Kinder („Das gefällt den Kindern sehr gut.“ (T2_4_1), „Und Kinder mutig wurden“ (T2_2_1) als wichtigen Referenzpunkt und zieht damit eher prozessorientierte Kriterien heran. Beim versierten Typ zeigt sich demgegenüber, dass die umgesetzten Methoden und Materialien dezidiert auf ihren zielführenden Charakter hin befragt und optimiert werden, ein Verweis auf die Outcome-Orientierung, wie hier am Beispiel der Forscherkisten erklärt wird:

[...] haben wir immer gemerkt, dass es nicht nur darum geht, das Material zu vervollständigen, sondern wirklich inhaltlich zu hinterfragen und wirklich zu überlegen, ist es genau das, was wir mit BiSS erreichen wollen. Oder ist es nicht dann auch eine typische Ansammlung von Material, die aber nicht genau das erfasst, was wir ja erreichen wollen. [...]. Nicht nur die Fachbegriffe, sondern eben auch die ganze Sprachbildung (T3_2_3).

Typenübergreifend wird auf generelle strukturelle Umsetzungsschwierigkeiten (v. a. unpassende Rahmenbedingungen, Personalmangel) verwiesen. Zudem wird die wahrgenommene Unverbindlichkeit der Fortbildungsinhalte problematisiert und auch die Sprachförderung selbst (,,wird uns ja immer wieder gesagt, dass wir frei sind in dem, was wir tun“" (T3_4_1) mit Blick auf deren alltagspraktische Implementierung und Verstetigung als mitunter erschwert betrachtet.

Die in den Fortbildungen angeregte Sensibilität und Reflexivität für das eigene Sprechen und das Anregen der Kinder zum Sprechen, die wie dargestellt typenübergreifend als eigener Lernerfolg markiert werden, verstetigt sich in der Praxis nicht automatisch. Vielmehr werden sie in den Alltagsroutinen als fragil erlebt. Eine stetige Begleitung durch kollegiale Hospitation und Beratung etwa wird daher als sehr wichtig erachtet, das Gelernte auch nachhaltig in der Praxis anwenden zu können.

Zusammenführung: Die berichteten Wirkungen von Fortbildungen stellen sich vor dem Hintergrund von Kita- (didaktischer Typ) bzw. Schulzentrierung (versierter Typ) als kontrastreich dar. Die Gegenüberstellung zeigt auf, dass Zufriedenheit und Akzeptanz, Lernerfolg bzw. Weiterentwicklung von Wissen und Können sowie Handeln und Performanz zentral durch die zugrundliegenden Handlungsorientierungen gerahmt sind. Während beim didaktischen Typ ein eher kindzentriertes sowie situations- und alltagsorientiertes Sprachförderhandeln ihre Perspektive auf (wirkungsvolle) Fortbildungen und darauf aufbauender Sprachförderung rahmt, wird beim versierten Typ eine an spezifischen Förderzielen orientierte zielgerichtete und planvolle Outcome-Orientierung den Deutungen zugrunde gelegt.

\section{Diskussion}

Um die Entwicklung der Sprachförderkompetenz der Fach- und Lehrkräfte zu messen, wurden aus dem Test SprachKoPF die beiden Skalen zum anwendungsbezogenen Wissen und zum Können herangezogen. Inwieweit diese Variablen verän- 
derungssensitiv für Zuwächse aufgrund von Fortbildungen sind, kann mit dieser Studie nicht geklärt werden, vor allem weil nicht geprüft werden konnte, inwieweit sich Fortbildungsinhalte und erfasste Kompetenzen im SprachKoPF decken. Dies ließ sich beispielsweise in der BiSS-Studie TRIO zeigen (Schierbaum et al. 2020). Die Entwickler*innen des Tests selbst (Tracy et al. 2012) berichten, dass sie keine Interaktionseffekte zwischen Fortbildungsbesuch und Förderkompetenzen feststellen konnten. Lediglich bei einer langen Dauer von Fortbildungen über $88 \mathrm{~h}$ gab es Zusammenhänge mit den Förderkompetenzen sowie bei Befragten mit einem hohen formalen Bildungsabschluss. Dies deckt sich mit den hier berichteten Ergebnissen: Fach- und Lehrkräfte mit einer großen Anzahl von besuchten Fortbildungen (Typ 2 und 3) und Lehrkräfte, die durch ihr Studium über einen hohen formalen Bildungsabschluss verfügen (Typ 4), haben größere Kompetenzwerte.

Die Befunde zu der ersten Forschungsfrage, wie sich Fach- und Lehrkräfte entlang ihrer handlungsleitenden Orientierungen hinsichtlich ihrer Sprachförderkompetenzen und ihres Fortbildungsbesuchs unterscheiden, legen nahe, dass die Differenzlinie nicht nur entlang der Berufsgruppen verläuft, sondern auch entlang des Umfangs an besuchten Fortbildungen und des Involviertseins in die Sprachförderung. Das Spektrum dessen, was die Teilnehmenden angaben, in den Fortbildungen gelernt zu haben, ist breit, auch wenn vergleichsweise wenige Fortbildungen besucht wurden, und zeigt, dass Fortbildungsinhalte teilweise großzügig interpretiert werden. Damit bildet die herangezogene Stichprobe die große Heterogenität ab, wie sie in vielen Studien benannt wird (vgl. Kap. 2) und dies, obwohl sich das hier berücksichtigte Sample ,lediglich“ auf 27 Personen bezieht. Die berichtete Breite des Qualifizierungsangebots zeigt deutlich auf, dass (bildungspolitische) Forderungen nach mehr Qualifizierungen für eine bessere Sprachförderung nicht pauschal zu unterstützen sind und Aussagen dazu, welche Wirkungen „die“ Fortbildungen erzielen, im Kontext Sprachförderung (am Übergang) schwerlich zu treffen sind. Gleichwohl, dies macht auch der Forschungsstand deutlich, gibt es empirische Hinweise auf die Wirksamkeit von Fortbildungen auf unterschiedlichen Ebenen.

Es gibt keine Angaben dazu, wie die Ankreuzliste im SprachKoPF zu den Selbsteinschätzungen, was man in den Fortbildungen gelernt hat, zustande kam. Die dort benannten Wissens- und Inhaltsbereiche konnten dem Spektrum von Fortbildungsthemen, wie sie im Fachdiskurs als relevant benannt werden (vgl. Kap. 2.2), zugeordnet werden. Aber ob das im Test operationalisierte Konstrukt Sprachförderkompetenz solche Fortbildungsinhalte in der von den Befragten verstandenen Weise abbildet, bleibt unklar.

Überzeugungen werden im Test nicht erfasst, was das festgestellte Desiderat bestätigt, dass das Wissen und Können im Vordergrund steht (vgl. Kap. 2.2). Somit kann mit den SprachKoPF-Daten wenig darüber ausgesagt werden, wie die Teilnehmenden die Fortbildungsinhalte aufgenommen haben und für ihre Sprachförderung mit den Kindern konkret nutzen. Deshalb wurde für weitere Analysen auf die beiden Sprachfördertypen fokussiert, die in ihren Einrichtungen stark in die Sprachförderung involviert sind, an vielen Fortbildungen zum Thema teilgenommen haben und im SprachKoPF hohe Testwerte erreicht haben, um zu erfahren, wie sie die Inhalte der Fortbildungen umsetzen, welche handlungsleitenden Orientierungen dem zugrundeliegen und wie sich die beiden Typen darin unterscheiden, auch weil sie un- 
terschiedlichen Berufsgruppen angehören. Die Befunde aus den Interviews weisen darauf hin, dass die Fortbildungen - trotz kaum messbarer Zuwächse beim Sprach$\mathrm{KoPF}$ - (selbstberichtete) Lernerfolge hervorbringen, worin diese bestehen und, dass diese Lernerfolge für die Typen sehr unterschiedlich ausfallen und dies, obwohl z. T. die gleichen Veranstaltungen im BiSS-Verbund besucht wurden.

Dazu konnten deutlich differenzierbare sowie typenübergreifende Deutungen auf allen drei Wirkungsebenen (Kirkpatrick und Kirkpatrick 2006) aufgezeigt werden (vgl. Forschungsfrage 2). Die qualitativ-explorative Rekonstruktion der Interviews erlaubt es nachzuvollziehen, unter welch spezifischen impliziten und habitualisierten Logiken Fortbildungsinhalte als bedeutsam und ertragreich eingeordnet und in die eigene Handlungspraxis überführt werden. Mit einem solchen Zugang kann ein Mehrwert in der Wirkungsforschung geschaffen werden, der - dies zeigen die Befunde - etwa darin liegt, vertiefte Erkenntnisse zum Label „Praxisrelevanz“ als bedeutsamem Parameter für die Wirkung von Fortbildungen zu liefern, indem diese als allgemeingültig anerkannten Gelingensfaktoren systematisch entlang von Typen ausdifferenziert werden. Zudem deuten die Ergebnisse darauf hin, dass es für die Gestaltung von (gemeinsamen) Fortbildungen relevant sein kann, Orientierungen und Kompetenzen stärker im Zusammenhang zu betrachten.

Als limitierend erweist sich, dass die konkreten (BiSS-)Fortbildungsinhalte nicht systematisch erfasst wurden. So konnte nicht geprüft werden, inwieweit die herangezogenen Items aus dem SprachKoPF diese Inhalte abbilden. Es wäre auch interessant, den typenspezifisch selektiven Blick auf Fortbildungen ins Verhältnis zu den „tatsächlichen“ Inhalten und Formaten zu setzen. Auch beschränken sich mit Fokus auf den kitabezogenen didaktischen und den schulzentrierten versierten Typ die Darlegungen an dieser Stelle auf eine berufsgruppenbezogene Kontrastierung, die Varianz innerhalb der Berufsgruppen wäre ebenso aufschlussreich; aus Platzgründen konnten die Analysen zum intuitiv-schematischen und zum konzeptionell-planvollen Typ nicht aufgenommen werden. Die systematischen Differenzen zwischen und zukünftig auch innerhalb der Berufsgruppen auf die Wirkungen von Fortbildungen sind auch deshalb von Bedeutung, da sich im Material zeigt, dass gemeinsame Fortbildungen im Kontext der Übergangsthematik zwar typenübergreifend als sehr fruchtbar erfahren werden, zugleich aber im Sprechen über das eigene Sprachförderhandeln doch stärker eine getrennte als eine gemeinsame Sprachförderpraxis zum Ausdruck kommt (Kämpfe und Betz 2020), wie sie den Ansätzen einer durchgängigen Sprachbildung als Kooperationserfordernis am Übergang (Salem 2018) eher zuwiderläuft.

Als limitierend erweist sich auch das Instrument SprachKoPF, obwohl damit ein standardisiertes Instrument zur Verfügung steht. Die Abneigung im Feld gegen Evaluation insgesamt und gegen diesen Onlinetest im Besonderen, führte zu so erheblichem Dropout, dass keine umfassenden statistischen Analysen möglich waren (zu hohen Dropouts in anderen Studien mit dem SprachKoPF: Schierbaum et al. 2020). Gleichzeitig erfassen die Items nur einen kleinen Ausschnitt der Sprachförderkompetenzen, die die Komplexität des Feldes und der Aufgabe nicht ausreichend abbilden.

Schließen möchten wir mit theoretischen Überlegungen, die im Kontext der sprachbezogenen Wirkungsforschung bislang unbeachtet blieben, aber interessantes 
Erklärungspotenzial sowie Anlass für weitere Forschung liefern. In professionstheoretischer Logik sollen Fortbildungen dazu verhelfen, „Einstellungen, Werthaltungen und Wissen (...) zu entwickeln, mit denen es möglich ist, in der Berufspraxis mit widersprüchlichen Handlungsanforderungen umzugehen“" (Von Hippel 2011, S. 250). Denn professionelles Handeln gilt als ,strukturelle[r] Ort der Vermittlung der eigentlich widerstreitenden Logiken von reflexiver, handlungsentlasteter Theorie und unter Handlungsdruck stehender Praxis“ (Helsper 2016, S. 54). Aus professionstheoretischer Perspektive wäre entsprechend zu erwarten, dass sich zwischen dem, was Fach- und Lehrkräfte unter gelingender Sprachförderung und entsprechenden Fortbildungen verstehen, und dem, was durch Politik, Fachwissenschaft und -praxis an Anforderungen an sie herangetragen wird (u. a. Betz et al. 2016) ein Spannungsfeld auftut (Bischoff 2017). Demgegenüber überrascht angesichts der sehr heterogenen Ausgangslagen, der eher ernüchternden Ergebnisse im SprachKoPF (Erdogan et al. 2021) und ebenso angesichts der berichteten strukturellen Schwierigkeiten in der Sprachförderung, die typenübergreifend weitgehende Zufriedenheit (Typ 1-4) mit den (praxisnahen) Fortbildungen sowie dem eigenen Sprachförderhandeln. In den Deutungen wird zugleich kaum ein Erwartungs- oder Legitimationsdruck (Begründungsantinomie; Helsper 2016) spürbar. Eine mögliche Erklärung dafür, dass sich solche Spannungsfelder in den Orientierungen der Interviewten nicht abbilden, könnte sein, dass diese sich gar nicht derart mit „einem von außen auferlegten „Anspruchszwang“““ (Helsper und Tippelt 2011, S. 277) konfrontiert sehen. Hierfür spricht, dass bildungspolitische Reformen, die von Initiativen wie BiSS begleitet werden, durch die Anforderung zur Umsetzung von (gelungener) Sprachförderung zwar einen Umsetzungsdruck erzeugen, das Wie und das zu erreichende Was aber weitgehend offenhalten. Das könnte, so eine vorsichtige Deutung, einen entlastenden Effekt haben und entsprechend auch den Fokus in den Orientierungen auch derjenigen mit geringen Fortbildungsvolumen auf unmittelbare praxisbezogene Fortbildung und deren Zufriedenheit mitbegründen. Inwiefern sich hierbei erneut differentielle i.S.v. typenspezifische Deutungsmuster unterscheiden lassen, wäre weiterer Forschung vorbehalten.

Funding Open Access funding enabled and organized by Projekt DEAL.

Open Access Dieser Artikel wird unter der Creative Commons Namensnennung 4.0 International Lizenz veröffentlicht, welche die Nutzung, Vervielfältigung, Bearbeitung, Verbreitung und Wiedergabe in jeglichem Medium und Format erlaubt, sofern Sie den/die ursprünglichen Autor(en) und die Quelle ordnungsgemäß nennen, einen Link zur Creative Commons Lizenz beifügen und angeben, ob Änderungen vorgenommen wurden.

Die in diesem Artikel enthaltenen Bilder und sonstiges Drittmaterial unterliegen ebenfalls der genannten Creative Commons Lizenz, sofern sich aus der Abbildungslegende nichts anderes ergibt. Sofern das betreffende Material nicht unter der genannten Creative Commons Lizenz steht und die betreffende Handlung nicht nach gesetzlichen Vorschriften erlaubt ist, ist für die oben aufgeführten Weiterverwendungen des Materials die Einwilligung des jeweiligen Rechteinhabers einzuholen.

Weitere Details zur Lizenz entnehmen Sie bitte der Lizenzinformation auf http://creativecommons.org/ licenses/by/4.0/deed.de. 


\section{Literatur}

Armstrong, K., Cusumano, D.L., Todd, M., \& Cohen, R. (2008). Literacy training for early childhood providers: changes in knowledge, beliefs, and instructional practices. Journal of Early Childhood Teacher Education, 29(4), 297-308.

Baumert, J., \& Kunter, M. (2006). Stichwort: Professionelle Kompetenz von Lehrkräften. Zeitschrift für Erziehungswissenschaft, 9(4), 469-520.

Beckerle, C. (2017). Alltagsintegrierte Sprachförderung im Kindergarten und in der Grundschule. Evaluation des „Fellbach-Konzepts“. Weinheim: Beltz Juventa.

Beher, K., \& Walter, M. (2012). Qualifikationen und Weiterbildung frühpädagogischer Fachkräfte. Bundesweite Befragung von Einrichtungsleitungen und Fachkräften in Kindertageseinrichtungen: Zehn Fragen - Zehn Antworten. Frühpädagogische Fachkräfte (WiFF Studien, Bd. 15). München: DJI.

Betz, T. (2013). Anforderungen an Fachkräfte in Kindertageseinrichtungen. In M. Stamm \& D. Edelmann (Hrsg.), Handbuch frühkindliche Bildungsforschung (S. 259-272). Wiesbaden: Springer VS.

Betz, T., Koch, K., Mehlem, U., \& Nentwig-Gesemann, I. (2016). Strukturwandel im Elementarbereich. Herausforderungen für pädagogische Fachkräfte und Organisationen am Beispiel des Umgangs mit Sprachförderung und Bildungsplänen. In K. Liebers, B. Landwehr, S. Reinhold, S. Riegler \& R. Schmidt (Hrsg.), Facetten grundschulpädagogischer und-didaktischer Forschung (S. 115-130). Wiesbaden: Springer VS.

Bischoff, S. (2017). Habitus und frühpädagogische Professionalität. Eine qualitative Studie zum Denken und Handeln von Fachkräften in Kindertageseinrichtungen. Weinheim: Beltz Juventa.

Böhm, J., Jungmann, T., \& Koch, K. (2017). Professionalisierung pädagogischer Fachkräfte. In T. Jungmann \& K. Koch (Hrsg.), Professionalisierung pädagogischer Fachkräfte in Kindertageseinrichtungen. Konzept und Wirksamkeit des KOMPASS-Projektes (S. 9-27). Wiesbaden: Springer VS.

Bohnsack, R. (2010). Rekonstruktive Sozialforschung. Einführung in qualitative Methoden (8. Aufl.). Leverkusen: Barbara Budrich.

Cloos, P., Schulz, M., \& Thomas, S. (2013). Wirkung professioneller Bildungsbegleitung von Eltern. Rekonstruktive Forschungsperspektiven auf kindheitspädagogische Settings. In L. Corell \& J. Lepperhoff (Hrsg.), Frühe Bildung in der Familie. Perspektiven der Familienbildung (S. 253-267). Weinheim: Beltz Juventa.

Egert, F., Fukkink, R., \& Eckhardt, A. G. (2018). Impact of in-service professional development programs for preschool teachers on quality ratings and child outcomes. Review of Educational Research, 88(3), 401-433.

Erdogan, E., Betz, T., Kämpfe, K., Kucharz, D., Mehlem, U., \& Rezagholinia, S. (2021). Dimensionen der Sprachförderung am Übergang Kindergarten-Grundschule. In S. Gentrup, S. Henschel, K. Schotte, L. Beck \& P. Stanat (Hrsg.), Sprach- und Schriftsprachförderung wirksam gestalten: Evaluation umgesetzter Konzepte (S. 84-104). Stuttgart: Kohlhammer.

Faas, S. (2013). Berufliche Anforderungen und berufsbezogenes Wissen von Erzieherinnen. Theoretische und empirische Rekonstruktionen. Wiesbaden: Springer.

Fröhlich-Gildhoff, K., Nentwig-Gesemann, I., \& Pietsch, S. (2011). Kompetenzorientierung in der Qualifizierung frühpädagogischer Fachkräfte (WiFF Expertisen). München: DJI.

Fukkink, R. G., \& Lont, A. (2007). Does training matter? A meta-analysis and review of caregiver training studies. Early Childhood Research Quarterly, 22(3), 294-311.

Hamre, B.K., Pianta, R.C., Burchinal, M., Field, S., LoCasale-Crouch, J., Downer, J.T., Howes, C., LaParo, K., \& Scott-Little, C. (2012). A course on effective teacher-child interactions: effects on teacher beliefs, knowledge, and observed practice. American Educational Research Journal, 49(1), 88-123.

Helsper, W. (2016). Antinomien und Paradoxien im professionellen Handeln. In M. Dick, W. Marotzki \& H. Mieg (Hrsg.), Handbuch Professionsforschung (S. 50-62). Bad Heilbrunn: Julius Klinkhardt.

Helsper, W., \& Tippelt, R. (2011). Ende der Profession und Professionalisierung ohne Ende? Zwischenbilanz einer unabgeschlossenen Diskussion. In W. Helsper \& R. Tippelt (Hrsg.), Pädagogische Professionalität (Zeitschrift für Pädagogik: Beiheft 57, S. 268-288). Weinheim: Beltz.

Von Hippel, A. (2011). Fortbildung in pädagogischen Berufen - zentrale Themen, Gemeinsamkeiten und Unterschiede der Fortbildung in Elementarbereich, Schule und Weiterbildung. In W. Helsper \& R. Tippelt (Hrsg.), Pädagogische Professionalität (Zeitschrift für Pädagogik: Beiheft 57, S. 248-267). Weinheim: Beltz.

Hopp, H., Thoma, D., \& Tracy, R. (2010). Sprachförderkompetenz pädagogischer Fachkräfte. Zeitschrift für Erziehungswissenschaft, 13(4), 609-629. 
Kammermeyer, G. (2019). Erfolgreiche Sprachbildung und Sprachförderung in Kitas braucht gute Fortbildung. Frühe Bildung, 8(4), 231-233.

Kammermeyer, G., Metz, A., Leber, A., Roux, S., Biskup-Ackermann, B., \& Fondel, E. (2019). Wie wirken sich Weiterbildungen auf die Anwendung von Sprachförderstrategien von pädagogischen Fachkräften in Kitas aus? Frühe Bildung, 8(4), 212-222.

Kämpfe, K., \& Betz, T. (2020). Wie Fach- und Lehrkräfte die deutsche Sprache am Übergang KitaGrundschule fördern. In N. Skorsetz, M. Bonanati \& D. Kucharz (Hrsg.), Diversität und soziale Ungleichheit. Herausforderungen an die Integrationsleistung der Grundschule (Jahrbuch Grundschulforschung, Bd. 24, S. 185-189). Wiesbaden: Springer VS.

Kirkpatrick, D.L., \& Kirkpatrick, J.D. (2006). Evaluating training programs: the four levels (3. Aufl.). San Francisco: Berrett-Koehler.

Kohler, B., \& Wacker, A. (2013). Das Angebots-Nutzungs-Modell. Überlegungen zu Chancen und Grenzen des derzeit prominentesten Wirkmodells der Schul- und Unterrichtsforschung. Die deutsche Schule, 105(3), 241-257.

Kucharz, D. (2018). Qualifizierung der Fachkräfte im Elementarbereich. In C. Titz, S. Geyer, H. Wagner, S. Weber \& M. Hasselhorn (Hrsg.), Konzepte zur Sprach- und Schriftsprachförderung entwickeln. Bildung durch Sprache und Schrift (S. 249-261). Stuttgart: Kohlhammer.

Kunter, M., \& Pohlmann, B. (2015). Lehrer. In E. Wild \& J. Möller (Hrsg.), Einführung in die Pädagogische Psychologie (S. 261-281). Berlin: Springer.

Kurucz, C., Hachfeld, A., Groeneveld, I., Roßbach, H.-G., \& Anders, Y. (2020). Überzeugungen und Selbstwirksamkeitserwartungen im Umgang mit kultureller Diversität in ihrem Zusammenspiel mit sprachpädagogischen Förderstrategien. Zeitschrift für Erziehungswissenschaft, 23(4), 709-738.

Lipowsky, F. (2019). Wie kommen Befunde der Wissenschaft ins Klassenzimmer? Impulse der Fortbildungsforschung. In C. Donie, F. Foerster, M. Obermayr, A. Deckwerth, G. Kammermeyer, G. Lenske, M. Leuchter \& A. Wildemann (Hrsg.), Grundschulpädagogik zwischen Wissenschaft und Transfer (S. 144-161). Wiesbaden: Springer VS.

Lipowsky, F., \& Rzejak, D. (2018). Empirische Befunde zur Wirksamkeit von Fortbildung für Lehrkräfte. In P. Platzbecker \& B. Priebe (Hrsg.), Zur Wirksamkeit und Nachhaltigkeit von Lehrerfortbildung. Qualitätssicherung und Qualitätsentwicklung katholischer Lehrerfort- und Weiterbildung (S. 34-74). Essen: Institut für Lehrerfortbildung.

Mackowiak, K., Beckerle, C., Koch, K., von Dapper-Saalfels, T., Löffler, C., Heil, J., \& Pauer, I. (2021). Kompetenzerwerb von pädagogischen Fachkräften in alltagsintegrierter Sprachförderung. Analysen aus dem ,,allE“-Projekt. In S. Gentrup, S. Henschel, K. Schotte, L. Beck \& P. Stanat (Hrsg.), Sprachund Schriftsprachförderung gestalten: Evaluation von Qualität und Wirksamkeit umgesetzter Konzepte (S. 66-83). Stuttgart: Kohlhammer.

Markussen-Brown, J., Juhl, C. B., Piasta, S. B., Bleses, D., Højen, A., \& Justice, L. M. (2017). The effects of language- and literacy-focused professional development on early educators and children: a bestevidence meta-analysis. Early Childhood Research Quarterly, 38(1), 97-115.

Ofner, D. (2014). Wie hängen Wissen und Handeln in der Sprachförderung zusammen? Eine explorative Untersuchung der Sprachförderkompetenz frühpädagogischer Fachkräfte. Empirische Pädagogik, 28(4), 302-318.

Redder, A., Schwippert, K., Hasselhorn, M., Forschner, S., Fickermann, D., Ehlich, K., Becker-Mrozeck, M., Krüger-Potratz, M., Roßbach, H.-G., Stanat, P., \& Weinert, S. (2011). Bilanz und Konzeptualisierung von strukturierter Forschung zu ,Sprachdiagnostik und Sprachförderung“. Zentrum zur Unterstützung der wissenschaftlichen Begleitung und Erforschung schulischer Entwicklungsprozesse (Bd. 2). https://epub.sub.uni-hamburg.de/epub/volltexte/2011/9874/pdf/zuse_berichte_02.pdf. Zugegriffen: 10. Febr. 2021.

Salem, T. (2018). Kooperation zwischen Kitas und Grundschulen für eine durchgängige Sprachbildung: Eine qualitative Fallvergleichsstudie. Münster: Waxmann.

Schierbaum, K., Kucharz, D., Brandenburg, J., Ehm, J.-H., Hasselhorn, M., Huschka, S. S., Geyer, S., Lausecker, A., Lemmer, R., \& Schulz, P. (2020). TRIO - Gemeinsame Qualifizierung des Fachpersonals in Grundschule und Kindertagesstätte zu alltagsintegrierter sprachlicher Bildung und Sprachförderung in Kleingruppen. In C. Titz, S. Weber, H. Wagner, A. Ropeter, S. Geyer \& M. Hasselhorn (Hrsg.), Sprach- und Schriftsprachförderung wirksam gestalten: Innovative Konzepte und Forschungsimpulse (S. 110-128). Stuttgart: Kohlhammer.

Schmidt-Hertha, B., \& Tippelt, R. (2011). Typologien. Report-Zeitschrift für Weiterbildungsforschung, 34(1), 23-35.

Schneider, W., Baumert, J., Becker-Mrotzek, M., Hasselhorn, M., Kammermeyer, G., Rauschenbach, T., Roßbach, H.-G., Roth, H.-J., Rothweiler, M., \& Stanat, P. (2012). Expertise „Bildung durch Sprache 
und Schrift (BiSS)“: Bund-Länder-Initiative zur Sprachförderung, Sprachdiagnostik und Leseförderung. https://www.biss-sprachbildung.de/ueber-biss/biss-expertise/. Zugegriffen: 10. Feb. 2021.

Schrader, J., Anders, Y., \& Richter, D. (2019). Fortbildung des pädagogischen Personals in der frühen Bildung, der Schule und der Erwachsenen- und Weiterbildung. In O. Köller, M. Hasselhorn, F. W. Hesse, K. Maaz, J. Schrader, H. Solga, C. K. Spieß \& K. Zimmer (Hrsg.), Das Bildungswesen in Deutschland (S. 775-802). Bad Heilbrunn: Julius Klinkhardt.

Simon, S., \& Sachse, S. (2011). Sprachförderung in der Kindertagesstätte - Verbessert ein Interaktionstraining das sprachförderliche Verhalten von Erzieherinnen? Empirische Pädagogik, 25(4), 462-480.

Stamm, M. (2014). Theoretische und empirische Konturen der internationalen kindheitspädagogischen Professionsforschung. In T. Betz \& P. Cloos (Hrsg.), Kindheit und Profession. Konturen und Befunde eines Forschungsfeldes (S. 116-129). Weinheim: Beltz Juventa.

Thoma, D., Ofner, D., \& Tracy, R. (2014). SprachKoPF-Online v072. Instrument zur standardisierten Erhebung der Sprachförderkompetenz pädagogischer Fachkräfte. Mannheim: Universität Mannheim.

Tracy, R., Thoma, D., Ofner, D., Michl, M., \& Seybel, C. (2012). Schlussbericht des Vorhabens SprachKoPF - Sprachliche Kompetenzen Pädagogischer Fachkräfte. Mannheim: Universität. https:// docplayer.org/32879585-Sprachkopf-sprachliche-kompetenzen-paedagogischer-fachkraefte.html. Zugegriffen: 8. Mai 2021.

Viernickel, S., Nentwig-Gesemann, I., Nicolai, K., Schwarz, S., \& Zenker, L. (2013). Schlüssel zu guter Bildung, Erziehung und Betreuung. Bildungsaufgaben, Zeitkontingente und strukturelle Rahmenbedingungen in Kindertageseinrichtungen. Berlin: Der Paritätische Gesamtverband, Diakonie Deutschland, Gewerkschaft Erziehung und Wissenschaft.

Vomhof, B. (2016). Frühpädagogische Fachkräfte und Eltern. Eine empirische Studie zu ihrer Zusammenarbeit im Kontext von Sprachfördermaßnahmen. Weinheim: Beltz Juventa.

Wirts, C., Cordes, A.-K., Egert, F., Fischer, S., Kappauf, N., Radan, J., Quehenberger, J., Danay, E., Dederer, V., \& Becker-Stoll, F. (2019). Abschlussbericht der Evaluationsprojekte BiSS-E1 und BiSS-E2. Wissenschaftliche Begleitung im Rahmen der Bund-Länder-Initiative Bildung durch Sprache und Schrift. München: Staatsinstitut für Frühpädagogik. https://www.ifp.bayern.de/projekte/qualitaet/ biss-e.php. Zugegriffen: 10. Febr. 2021. 